\title{
Energy Optimization via Process Modification to Maximize Economic Feasibility of the Butane Gas Splitting Process
}

Yeongryeol Choil,2, Nahyeon An³, II Moon', Junghwan Kim², *

${ }^{1}$ Department of Chemical and Biomolecular Engineering, Yonsei University, 50 Yonsei-ro, Seodaemun-gu, Seoul 03722, Republic of Korea

${ }^{2}$ Green Materials and Processes R\&D Group, Korea Institute of Industrial Technology, 55 Jongga-ro, Jung-gu, Ulsan 44413, Republic of Korea

${ }^{3}$ School of Energy and Chemical Engineering, Ulsan National Institute of Science and Technology (UNIST), 50 UNIST-gil, Eonyang-eup, Ulju-gun, Ulsan 44919, Republic of Korea

(kjh31@kitech.re.kr)

\section{Supporting Information}




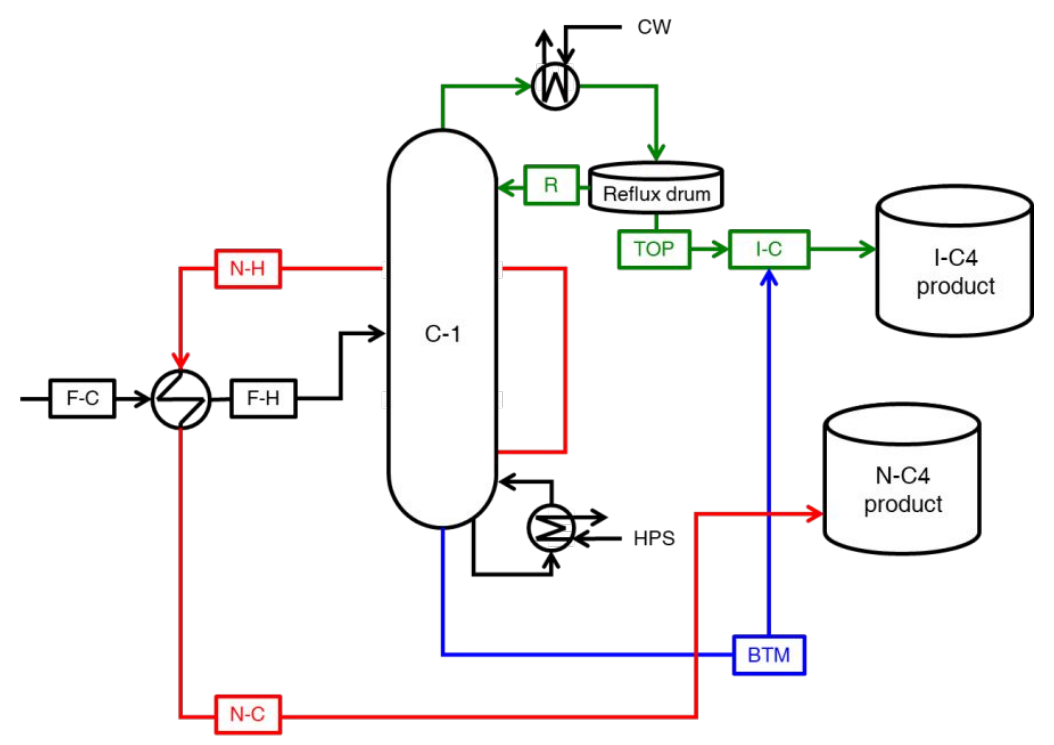

Figure S1. Process diagram for one column

Table S1. Column design conditions

\begin{tabular}{lllll}
\hline Name & Unit & Case 1 & Case 2 & Case 3 \\
\hline Propane & $\mathrm{wt} . \%$ & 0.91 & 0.43 & 0.10 \\
i-butane & $\mathrm{wt} . \%$ & 30.58 & 18.55 & 2.99 \\
n-butane & $\mathrm{wt} \% \%$ & 66.77 & 80.33 & 96.73 \\
1-butene & $\mathrm{wt} . \%$ & 0.00 & 0.05 & 0.00 \\
i-butene & $\mathrm{wt.} \%$ & 0.00 & 0.15 & 0.00 \\
t-2-butene & $\mathrm{wt} \% \%$ & 0.00 & 0.10 & 0.12 \\
c-2-butene & $\mathrm{wt} . \%$ & 0.00 & 0.05 & 0.04 \\
n-pentane & $\mathrm{wt} . \%$ & 0.37 & 0.17 & 0.02 \\
i-pentane & $\mathrm{wt} . \%$ & 1.37 & 0.17 & 0.00 \\
Water & $\mathrm{wt} . \%$ & 0.00 & 0.00 & 0.00 \\
Mass flow & $\mathrm{kg} / \mathrm{h}$ & 4313.65 & 3613.45 & 3038.50 \\
Feedstock temperature & ${ }^{\circ} \mathrm{C}$ & 45.80 & 45.80 & 45.80 \\
Feedstock pressure & $\mathrm{kg} / \mathrm{cm} 2 \mathrm{~g}$ & 7.70 & 7.70 & 7.70 \\
Temperature of TOP & ${ }^{\circ} \mathrm{C}$ & 57.00 & 61.00 & 58.00 \\
Temperature of BTM & ${ }^{\circ} \mathrm{C}$ & 95.00 & 85.00 & 74.00 \\
Pressure of TOP & $\mathrm{kg} / \mathrm{cm} 2 \mathrm{~g}$ & 7.30 & 7.30 & 7.30 \\
Pressure of BTM flow & $\mathrm{kg} / \mathrm{cm} 2 \mathrm{~g}$ & 7.90 & 7.90 & 7.90 \\
\hline
\end{tabular}


Table S2. Validation results for case 1

\begin{tabular}{|c|c|c|c|c|c|c|c|c|c|c|}
\hline \multirow[t]{2}{*}{ Name } & \multirow[t]{2}{*}{ Unit } & \multicolumn{3}{|c|}{ n-butane product flow $(\mathrm{N}-\mathrm{H})$} & \multicolumn{3}{|c|}{ i-butane product flow (TOP) } & \multicolumn{3}{|c|}{ Bottom flow (BTM) } \\
\hline & & Design & Simulation & Difference & Design & Simulation & Difference & Design & Simulation & Difference \\
\hline Propane & wt. $\%$ & 0.00 & 0.00 & 0.00 & 2.98 & 2.98 & 0.00 & 0.00 & 0.00 & 0.00 \\
\hline i-Butane & wt. $\%$ & 2.90 & 3.25 & 0.35 & 93.83 & 93.05 & -0.78 & 0.00 & 0.02 & 0.02 \\
\hline n-Butane & wt.\% & 96.01 & 96.00 & -0.01 & 3.19 & 3.97 & 0.78 & 26.48 & 9.42 & -17.06 \\
\hline 1-Butene & wt.\% & 0.00 & 0.00 & 0.00 & 0.00 & 0.00 & 0.00 & 0.00 & 0.00 & 0.00 \\
\hline i-Butene & wt. $\%$ & 0.00 & 0.00 & 0.00 & 0.00 & 0.00 & 0.00 & 0.00 & 0.00 & 0.00 \\
\hline t-2-Butene & wt.\% & 0.00 & 0.00 & 0.00 & 0.00 & 0.00 & 0.00 & 0.00 & 0.00 & 0.00 \\
\hline c-2-Butene & wt.\% & 0.00 & 0.00 & 0.00 & 0.00 & 0.00 & 0.00 & 0.00 & 0.00 & 0.00 \\
\hline n-Pentane & wt.\% & 0.22 & 0.14 & -0.08 & 0.00 & 0.00 & 0.00 & 16.62 & 20.51 & 3.89 \\
\hline i-Pentane & wt. $\%$ & 0.87 & 0.61 & -0.26 & 0.00 & 0.00 & 0.00 & 56.90 & 70.05 & 13.15 \\
\hline Water & wt. $\%$ & 0.00 & 0.00 & 0.00 & 0.00 & 0.00 & 0.00 & 0.00 & 0.00 & 0.00 \\
\hline Mass flow & $\mathrm{kg} / \mathrm{h}$ & 2940.05 & 2940.05 & 0.00 & 1315.25 & 1315.25 & 0.00 & 58.35 & 58.35 & 0.00 \\
\hline Temperature & ${ }^{\circ} \mathrm{C}$ & 73.00 & 73.00 & 0.00 & 51.00 & 51.00 & 0.00 & 95.00 & 95.00 & 0.00 \\
\hline Pressure & $\mathrm{kg} / \mathrm{cm}^{2}$ & - & 7.79 & - & 7.30 & 7.30 & 0.00 & 7.90 & 7.90 & 0.00 \\
\hline
\end{tabular}


Table S3. Validation results for case 2

\begin{tabular}{lllllllllll}
\hline \multirow{2}{*}{ Name } & Unit & \multicolumn{2}{l}{ n-butane product flow (N-H) } & \multicolumn{2}{l}{ i-butane product flow (TOP) } & \multicolumn{3}{c}{ Bottom flow(BTM) } \\
\cline { 3 - 10 } & & Design & Simulation & Difference & Design & Simulation & Difference & Design & Simulation & Difference \\
\hline Propane & wt.\% & 0.00 & 0.00 & 0.00 & 2.36 & 2.38 & 0.01 & 0.00 & 0.00 & 0.00 \\
i-Butane & wt.\% & 2.04 & 2.25 & 0.20 & 93.32 & 92.39 & -0.93 & 0.00 & 0.07 & 0.07 \\
n-Butane & wt.\% & 97.34 & 97.34 & 0.00 & 4.32 & 4.60 & 0.28 & 64.69 & 55.93 & -8.76 \\
1-Butene & wt.\% & 0.06 & 0.03 & -0.03 & 0.00 & 0.15 & 0.15 & 0.00 & 0.00 & 0.00 \\
i-Butene & wt.\% & 0.18 & 0.08 & -0.10 & 0.00 & 0.48 & 0.48 & 0.00 & 0.01 & 0.01 \\
t-2-Butene & wt.\% & 0.13 & 0.12 & 0.00 & 0.00 & 0.01 & 0.01 & 0.00 & 0.08 & 0.08 \\
c-2-Butene & wt.\% & 0.07 & 0.06 & -0.01 & 0.00 & 0.00 & 0.00 & 0.00 & 0.08 & 0.08 \\
n-Pentane & wt.\% & 0.09 & 0.06 & -0.03 & 0.00 & 0.00 & 0.00 & 18.04 & 22.43 & 4.39 \\
i-Pentane & wt.\% & 0.10 & 0.07 & -0.03 & 0.00 & 0.00 & 0.00 & 17.27 & 21.41 & 4.14 \\
Water & wt.\% & 0.00 & 0.00 & 0.00 & 0.00 & 0.00 & 0.00 & 0.00 & 0.00 & 0.00 \\
Mass flow & $\mathrm{kg} / \mathrm{h}$ & 2940.00 & 2940.00 & 0.00 & 654.05 & 654.05 & 0.00 & 19.40 & 19.40 & 0.00 \\
Temperature & ${ }^{\circ} \mathrm{C}$ & 73.00 & 73.00 & 0.00 & 61.00 & 61.00 & 0.00 & 85.00 & 85.00 & 0.00 \\
Pressure & $\mathrm{kg} / \mathrm{cm}^{2}$ & - & 7.79 & - & 7.30 & 7.30 & 0.00 & 7.90 & 7.90 & 0.00
\end{tabular}


Table S4. Validation results for case 3

\begin{tabular}{lllllllllll}
\hline \multirow{2}{*}{ Name } & \multirow{2}{*}{ Unit } & \multicolumn{2}{l}{ n-butane product flow $(\mathrm{N}-\mathrm{H})$} & \multicolumn{3}{l}{ i-butane product flow (TOP) } & \multicolumn{3}{c}{ Bottom flow(BTM) } \\
\cline { 3 - 10 } & & Design & Simulation & Difference & Design & Simulation & Difference & Design & Simulation & Difference \\
\hline Propane & wt.\% & 0.00 & 0.00 & 0.00 & 3.37 & 3.41 & 0.04 & 0.00 & 0.00 & 0.00 \\
i-butane & wt.\% & 0.57 & 0.58 & 0.00 & 83.10 & 82.96 & -0.14 & 0.00 & 0.03 & 0.03 \\
n-butane & wt.\% & 99.25 & 99.25 & 0.00 & 13.53 & 13.61 & 0.08 & 96.52 & 95.73 & -0.79 \\
1-butene & wt.\% & 0.00 & 0.00 & 0.00 & 0.00 & 0.00 & 0.00 & 0.00 & 0.00 & 0.00 \\
i-butene & wt.\% & 0.00 & 0.00 & 0.00 & 0.00 & 0.00 & 0.00 & 0.00 & 0.00 & 0.00 \\
t-2-butene & wt.\% & 0.12 & 0.12 & 0.00 & 0.00 & 0.02 & 0.02 & 0.21 & 0.12 & -0.09 \\
c-2-butene & wt.\% & 0.04 & 0.04 & 0.00 & 0.00 & 0.00 & 0.00 & 0.11 & 0.09 & -0.02 \\
n-pentane & wt.\% & 0.01 & 0.01 & 0.00 & 0.00 & 0.00 & 0.00 & 3.16 & 4.03 & 0.86 \\
i-pentane & wt.\% & 0.00 & 0.00 & 0.00 & 0.00 & 0.00 & 0.00 & 0.00 & 0.00 & 0.00 \\
Water & wt.\% & 0.00 & 0.00 & 0.00 & 0.00 & 0.00 & 0.00 & 0.00 & 0.00 & 0.00 \\
Mass flow & $\mathrm{kg} / \mathrm{h}$ & 2940.00 & 2940.00 & 0.00 & 89.05 & 89.02 & -0.03 & 9.48 & 9.48 & 0.00 \\
Temperature & ${ }^{\circ} \mathrm{C}$ & 73.00 & 73.00 & 0.00 & 57.00 & 57.00 & 0.00 & 74.00 & 74.00 & 0.00 \\
Pressure & $\mathrm{kg} / \mathrm{cm}^{2}$ & - & 7.79 & - & 7.30 & 7.30 & 0.00 & 7.90 & 7.90 & 0.00 \\
\hline
\end{tabular}

